\title{
Analysis of the clinical effects of transforaminal endoscopic discectomy on lumbar disk herniation combined with common peroneal nerve paralysis: a 2-year follow-up retrospective study on 32 patients
}

This article was published in the following Dove Press journal: Journal of Pain Research

5 January 2017

Number of times this article has been viewed

\author{
Ya-peng Wang* \\ Wei Zhang \\ Jian Zhang \\ Ya-peng Sun* \\ ji-long An \\ Wen-yuan Ding
}

Department of Spinal Surgery, The Third Hospital of Hebei Medical University, Shijiazhuang, People's Republic of China

*These authors contributed equally to this work

Correspondence: Wei Zhang Department of Spinal Surgery, The Third Hospital of Hebei Medical University, Shijiazhuang, No. 139, Ziqiang Road, Shijiazhuang City 050000, Hebei Province, People's Republic of China

Tel/fax +86 3II 88602082

Email wypsmile866@I63.com
Background: Very few studies have discussed transforaminal endoscopic discectomy (TED) in the treatment of common peroneal nerve paralysis induced by lumbar disk herniation (LDH). This study aimed to evaluate the efficacy of TED in the treatment of LDH combined with common peroneal nerve paralysis.

Materials and methods: The clinical and follow-up data of 32 patients with common peroneal nerve paralysis induced by LDH undergoing TED from March 2011 to April 2014 were retrospectively analyzed in this study. Follow-up was conducted immediately after the surgery, as well as 3, 12, and 24 months postoperatively. The parameters (including muscle strength recovery of the anterior tibial muscle, leg pain visual analog scale score, neurological function Japanese Orthopaedic Association [JOA] score, MacNab scores in the last follow-up, and the intraoperative and postoperative complications) were recorded.

Results: Three patients $(9.4 \%)$ had the anterior tibial muscle strength recovered to $\geq$ grade 4 immediately after the surgery. The anterior tibial muscle strength of patients recovered to basically stable form in the 6-month postoperative follow-up and that in the last follow-up were as follows: one case of grade 1, one case of grade 2, 28 cases of grade 4, and two cases of grade 5 . The visual analog scale scores of leg pain were significantly reduced immediately after the surgery and also on 3, 12, and 24 months compared with preoperative period (all $P<0.05$ ). The postoperative JOA scores in the last follow-up were significantly higher than the preoperative JOA scores $(P<0.05)$, and there were nine excellent cases $(28.2 \%), 21$ good cases $(65.6 \%)$, one fair case $(3.1 \%)$ and one poor case $(3.1 \%)$ in the last follow-up, with an overall excellent and good rate of $93.8 \%$.

Conclusion: TED, which can offer sufficient decompression of the nerve root, has excellent overall clinical effects in treating common peroneal nerve paralysis induced by LDH.

Keywords: transforaminal endoscopic discectomy, lumbar disk herniation, common peroneal nerve paralysis, minimally invasive surgery

\section{Introduction}

Lumbar disk herniation (LDH) is the most common reason causing lumbocrural pain, which severely affects the life and work of patients. However, LDH-induced common peroneal nerve paralysis is relatively rare, so is the relevant clinical research. ${ }^{1-4}$ Common peroneal nerve paralysis will result in severe motor dysfunction in the lower extremities, which frequently requires surgical intervention. ${ }^{1}$ At present, the commonly 
used surgical procedure is open lumbar posterior nerve root decompression and exploration. ${ }^{2}$ However, the traditional open surgery is associated with large incision, requiring extensive stripping of the paravertebral soft tissues, heavy trauma, and a large amount of blood loss, which usually leads to denervation of muscle and atrophy, ${ }^{5-7}$ and in addition, the patients suffer from long-term postoperative lumbodorsal muscle pain. These postoperative complications seriously affect the clinical recovery and patient satisfaction.

In recent years, the thriving advances in the field of minimally invasive spine surgery rendered revolutionary progress in the spinal endoscopic technology. The percutaneous transforaminal endoscopic discectomy (TED) is becoming increasingly advanced, and it has been granted extensive importance as a result of its advantages such as more minimally invasive, more accurate, and rapid postoperative recovery. ${ }^{8,9}$ This has also provided new arenas for the surgical treatment of patients with LDH-induced common peroneal nerve paralysis. In 2016, Chun and Park ${ }^{10}$ reported the application of TED in treating one case with L5-S1 extreme lateral LDH-induced common peroneal nerve paralysis, whose anterior tibial muscle strength recovered to grade 5 after 6 weeks, postoperatively. However, there is no report regarding a large sample study on applying such procedure exclusively in LDH-induced common peroneal nerve paralysis yet, and hence its safety and effectiveness remain unclear.

This study retrospectively analyzed the clinical data of 32 patients with LDH-induced common peroneal nerve paralysis who received TED in our department. The study evaluated the safety and effectiveness of TED in treating LDH-induced common peroneal nerve paralysis. The study provided a reference for practical operation in clinical settings.

\section{Materials and methods}

\section{General data}

The clinical data of 35 cases of patients with LDH-induced common peroneal nerve paralysis who received TED in the Third Hospital of Hebei Medical University from March 2011 to April 2014 were retrospectively analyzed. Finally, 32 cases who met the inclusion and exclusion criteria were enrolled. The study was approved by the ethics committee of the Third Hospital of Hebei Medical University. Written informed consent was obtained from all patients and their families.

The inclusion criteria were degenerative changes in single segment of lumbar intervertebral disk accompanied with different degrees of unilateral lower limb nerve root pain, imaging manifestations being consistent with clinical symptoms and signs; no lumbar instability on the preoperative X-ray examination in lumbar dynamic position, and all patients with the characteristic symptom of unilateral foot drop. The diagnostic standard of common peroneal nerve paralysis ${ }^{4}$ was anterior tibial muscle strength of $\leq$ grade 3 .

The exclusion criteria were patients with obvious degenerative deformities, instability, disk calcification, lumbar spinal canal, and intervertebral foramen bony stenosis; patients with severe prolapse and dissociation of nucleus pulposus and with extreme lateral LDH; patients requiring revision surgery after the lumbar surgery; patients with cauda equina syndrome, lumbar foraminal and extraforaminal stenosis; pathological state such as concurrent infection, tumor, or fracture; the protruding segment that was proposed for surgery was not subjected to the iliac crest and L5 parapophysis; obese patients with body mass index of $\geq 28 \mathrm{~kg} / \mathrm{m}^{2}$ and finally common peroneal nerve paralysis caused by peripheral neuropathy.

All the data were collected by two physicians who did not take part in research design, analysis, interpretation, or decision-making of article publishing. The data were analyzed, and the results were interpreted by another physician who was blind to the data collection process.

\section{Surgical technique}

All the operations were performed by the same surgeon. The patient was placed in the lateral position with the affected side being placed upward and the uninjured side was blocked up. X-ray frontal fluoroscopic angle paralleled the lesion segment end plate before the surgery. The diseased intervertebral disk plane and the posterior midline of spinal process were marked. The entry point and side open distance depended on the patients' physique and different types of LDH. In general, the side open distance was $12-14 \mathrm{~cm}$.

The surgical approach was a lateral approach. Lidocaine (1\%) was injected for the anesthesia of skin and deep fascia after disinfection and draping. An 18-gauge spinal needle was inserted from the entry point and punctured under the guidance of $\mathrm{C}$-arm radiograph. The needlepoint reached the anterior inferior border of zygapophysis. A 22-gauge spinal needle was taken from the 18 -gauge spinal needle, and a guide wire was inserted into the needle. When the position was proper under fluoroscopic guidance, the spinal needle was taken out. Taking the entry point as the center, an $8 \mathrm{~mm}$ incision was made on the skin. The guide bar was inserted along with the guide wire, and the surgical channel was expanded through step-by-step expansion of the catheter. Followed by gradual removal of the dilating catheter, a serrated reamer was inserted along with the guide bar. Partial sclerotin on the outer edges of superior zygapophysis was removed under 
the guidance of C-arm radiograph. It was important that the surgeon expand the intervertebral foramen toward the dorsal side of the dural sac consciously. Particular attention was paid that the serrated reamer never exceeded the inner edge of pedicle of vertebral arch to protect the dura mater and nerve from injury. After removing the trephine, the working casing was placed along with the guide bar. When the position was proper according to fluoroscopic guidance, the endoscope was placed. The protruding disk and its debris were observed through the endoscope. The degenerative nucleus pulposus was extracted using the proprietary nucleus pulposus forceps through the TED channel. Clearly visible dura under endoscope and pulsation with the heart beat were the significant indicators of thorough decompression and termination of the surgery. Crinkle plasty was performed on tear opening of fiber ring with radio frequency bipolar electrode. Coagulation hemostasis was used to stop the bleeding within the surgical field. The working casing was rotated to examine whether bleeding and debris were present within the surgical field and the endoscope and working casing were removed after confirming that there was no abnormality. The incision was sutured with one stitch.

\section{Postoperative treatment}

The patients were in repose for 1-2 days postoperatively and ambulated under supervision after wearing orthosis equipment. Once stable, patients were assisted with muscle strengthening exercise and straight leg raising exercises. After 1 week, patients were allowed to perform some light manual labor. Treatment with dehydration, hemostasis, and nutritional nerve agents was administered in accordance with specific conditions.

\section{Clinical assessment Functional assessment}

The visual analog scale (VAS) was adopted to assess the leg pain 1 day prior to surgery, immediately after surgery, as well as 3, 12, and 24 months after surgery. The Japanese Orthopaedic Association (JOA) score was adopted to assess the neurological function of patients 1 day before surgery, as well as 12 and 24 months after surgery. The anterior tibial muscle strength of patients was measured immediately after surgery, as well as during the follow-up at 3, 6, 12, and 24 months postoperatively, until the anterior tibial muscle strength recovered to the stable level, which was also considered as the postoperative recovery level. When the postoperative anterior tibial muscle strength recovered to grade 4 or 5 , the patients could have the dorsal stretch of the ankle, and the gait basically returned to the healed state. The patients who could not achieve the abovementioned standards were designated as unhealed. The patients whose anterior tibial muscle strength recovered to grade 5 were labeled as completely healed, while those patients who recovered to grade 4 were labeled as incompletely healed. The course of foot drop, preoperative anterior tibial muscle strength, age, sex, sensory disorder in the affected extremity, and decompressed nerve root between the healed and unhealed patients were compared to determine the factors that might influence the postoperative recovery of patients.

\section{Imaging assessment}

The lumbar X-ray radiographs in the anteroposterior and lateral position as well as dynamic position, lumbar intervertebral disk computed tomography and lumbar magnetic resonance imaging (MRI) were obtained preoperatively. The lumbar X-ray radiographs in the dynamic position were obtained immediately after surgery, as well as 3 months after surgery, to observe whether there was any intervertebral instability. The lumbar MRI was conducted 3 months postoperatively to observe whether the nerve root compression was relieved or has any recurrence.

\section{Adverse events}

The adverse events included postoperative revision, complications (other complications such as partial residue of the nucleus pulposus, dural laceration, nerve injury, postoperative intervertebral space infection, retroperitoneal hematoma, ${ }^{11}$ postoperative paresthesia, ${ }^{12}$ abdominal organ injury, epilepsy, ${ }^{13}$ and postoperative pseudocyst ${ }^{14}$ ), as well as death within 2 weeks postoperatively.

\section{Other assessments}

Other perioperative data included surgical segment, estimated amount of operative bleeding (milliliters), duration of operation (minutes), as well as intraoperative duration of fluoroscopy (time) and the medical resources used (postoperative length of hospital stay and the direct medical expenses brought by surgery, hospitalization, and other surgical operations).

\section{Statistical analysis}

The SPSS 19.0 software package was adopted for statistical analysis. Qualitative data were expressed as number or percentage. Quantitative data were expressed in mean \pm standard deviation or median (P25, P75) as appropriate. Wilcoxon signed-rank test was applied for comparing the postoperative follow-up and preoperative VAS scores. The paired $t$-test was performed to analyze the preoperative and 
postoperative JOA scores of patients. A $P$-value of $<0.05$ was considered statistically significant.

\section{Results}

\section{Baseline demographic data and operation condition}

All the surgeries were performed smoothly, and the complete data of 32 cases were included in this research. The baseline demographic data of patients are shown in Table 1.

\section{Clinical outcomes JOA scores}

The neurological function JOA scores immediately after surgery as well as at 3,12, and 24 months after surgery were remarkably increased relative to those before surgery (all $P<0.05$; Table 2).

Table I Baseline demographic data and operation of patients

\begin{tabular}{|c|c|}
\hline Item & $\begin{array}{l}\text { Transforaminal endoscopic } \\
\text { discectomy }(\mathrm{N}=32)\end{array}$ \\
\hline Age $(\bar{x} \pm s$, years $)$ & $48.7 \pm 8.6$ \\
\hline \multicolumn{2}{|l|}{ Sex (\%) } \\
\hline Male & $18(56.25)$ \\
\hline Female & $14(43.75)$ \\
\hline \multicolumn{2}{|l|}{ Surgical segment (\%) } \\
\hline $\mathrm{L} 3 / 4$ & $5(15.62)$ \\
\hline $\mathrm{L} 4 / 5$ & $19(59.38)$ \\
\hline L5/SI & $8(25.00)$ \\
\hline $\begin{array}{l}\text { Preoperative course of disease } \\
(\bar{x} \pm s, \text { months })\end{array}$ & $10.2 \pm 6.1$ \\
\hline Complication (case) ${ }^{\mathrm{a}}$ & 9 \\
\hline $\begin{array}{l}\text { Intraoperative blood loss volume } \\
(\bar{x} \pm s, \mathrm{~mL})\end{array}$ & $80.5 \pm 15.0$ \\
\hline $\begin{array}{l}\text { Duration of operation }(\bar{x} \pm s \text {, } \\
\text { minutes) }\end{array}$ & $124.5 \pm 23.5$ \\
\hline $\begin{array}{l}\text { Times of intraoperative } \\
\text { fluoroscopy }(\bar{x} \pm s, \text { time })\end{array}$ & $22.0 \pm 6.0$ \\
\hline Length of stay $(\bar{x} \pm s$, days $)$ & $3.2 \pm 1.0$ \\
\hline Surgical expenses $(\bar{x} \pm s, ¥ \mid 0,000)$ & $2.5 \pm 0.4$ \\
\hline $\begin{array}{l}\text { Postoperative follow-up time } \\
(\bar{x} \pm s, \text { months })\end{array}$ & $24.0 \pm 0.0$ \\
\hline Postoperative revision (case) & 0 \\
\hline Postoperative complication (case) ${ }^{\mathrm{b}}$ & 2 \\
\hline
\end{tabular}

\section{VAS scores}

The radiating pain VAS scores immediately after surgery as well as at 3,12 , and 24 months after surgery were notably decreased compared with those before surgery (all $P<0.05$; Table 2).

\section{Anterior tibial muscle strength}

Three patients had the anterior tibial muscle strength recovered to grade 4 immediately after the surgery, which basically recovered to stable level in the 6 months after the surgery. The anterior tibial muscle strength of patients in the last follow-up was shown as follows: one case of grade 1 , one case of grade 2, 28 cases of grade 4, and two cases of grade 5 , with the overall recovery rate of $93.8 \%$ (30/32).

\section{MacNab scores}

There were nine excellent cases $(28.2 \%), 21 \operatorname{good}(65.6 \%)$, one fair $(3.1 \%)$, and one poor (3.1\%) in the last follow-up, with the overall excellent and good rate of $93.8 \%$.

\section{Imaging findings}

The lumbar X-ray reexamination in dynamic position was conducted on all patients 3 months postoperatively, and no intervertebral instability was found. The lumbar MRI reviewed 3 months postoperatively was compared with MRI taken preoperatively, which indicated that the protruding intervertebral disk significantly rebounded and disappeared, and no recurrence in the surgical segment was seen.

\section{Complications}

After surgery, two patients developed postoperative complications. A single case developed dural injury. The patient was administered the symptomatic treatments of fluid infusion (500 $\mathrm{mL}$ of $0.9 \%$ sodium chloride injection and $500 \mathrm{~mL}$ of $5.0 \%$ glucose injection) and antibiotic for preventing infection $(1.0 \mathrm{~g}$ of ceftriaxone for intravenous infusion twice a day). The patient healed 2 weeks after surgery without symptoms such as hypotensive cranial pressure headache, and the incision showed signs of primary healing. The remaining one case developed abnormal sensation in export nerve root

Table 2 The preoperative and postoperative JOA scores and VAS scores

\begin{tabular}{lllllll}
\hline & Preoperative & Immediately after the surgery & 3 months & I 2 months & 24 months & P-Value \\
\hline JOA & $13.7 \pm 0.8$ & $18.5 \pm 0.5$ & $20.0 \pm 0.3$ & $21.5 \pm 0.6$ & $22.5 \pm 0.7$ & $<0.05$ \\
VAS & $8(7,8)$ & $3(I, 3)$ & $2(I, 2)$ & $1(1,2)$ & $1(I, 2)$ & $<0.05$ \\
\hline
\end{tabular}

Notes: Data presented as $\bar{x} \pm s$ or median (quartiles).

Abbreviations: JOA, Japanese Orthopaedic Association; VAS, visual analog scale. 
zone on the second day after the surgery, which disappeared after 3 days of symptomatic treatment with dehydration $(50 \mathrm{~g}$ intravenous injection of mannitol once daily) and administration of hormone $(10 \mathrm{mg}$ of dexamethasone for intravenous drip once daily).

\section{Typical case}

Figures 1 and 2 show the radiographs and MRI, respectively, of a representative 48-year-old male patient with left lower extremity pain accompanied with common peroneal nerve paralysis who was treated with TED (Figure 3).
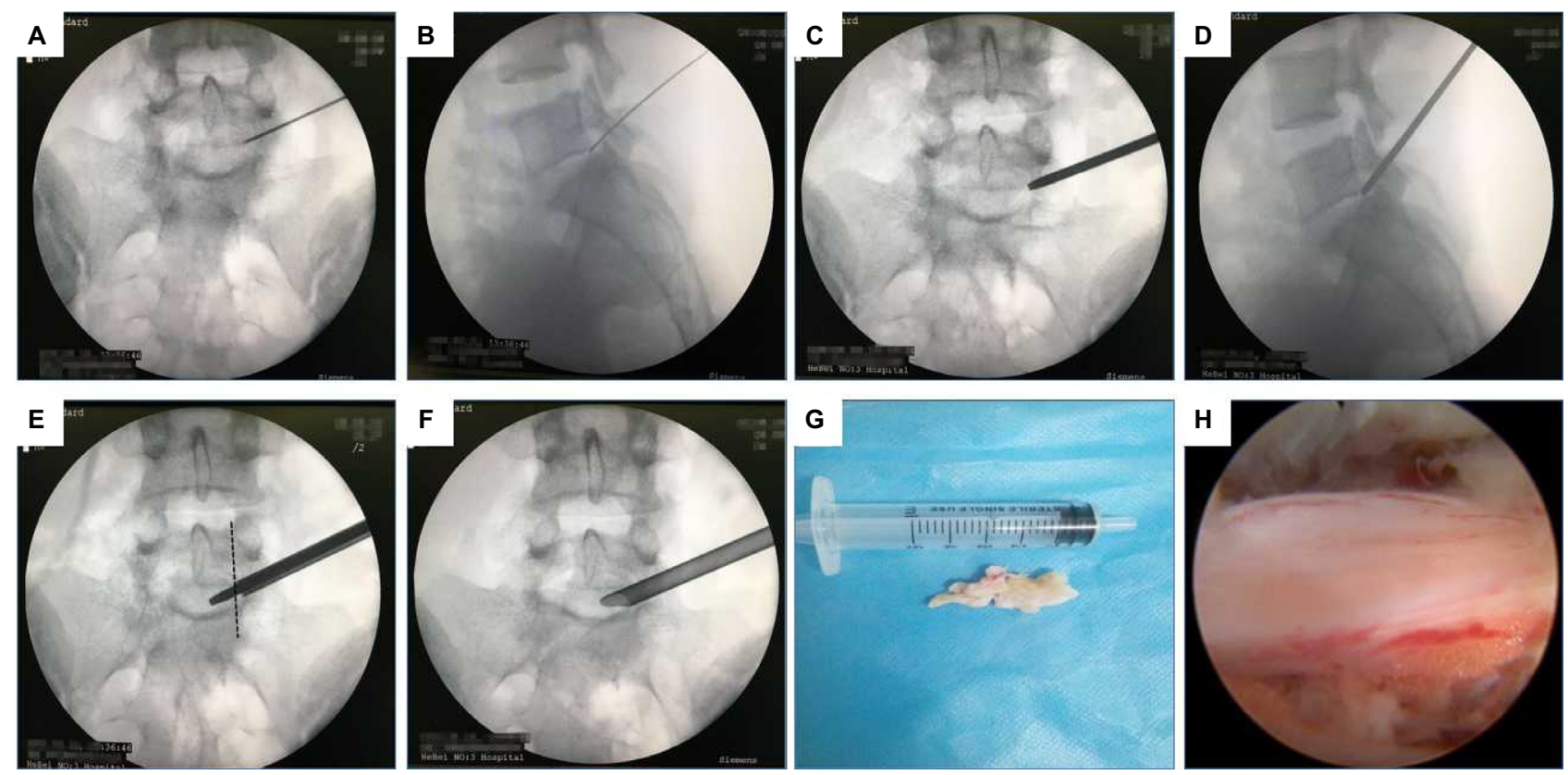

Figure I A case of transforaminal endoscopic discectomy surgery.

Notes: Anteroposterior and lateral position X-ray radiographs indicate the location of the spinal needle ( $\mathbf{A}$ and $\mathbf{B})$; anteroposterior and lateral position $\mathbf{X}$-ray radiographs indicate the location of the guide bar ( $\mathbf{C}$ and $\mathbf{D})$; the serrated reamer was utilized for zygapophyseal plasty, and the front end of the serrated reamer should not exceed the inner edge of pedicle of vertebral arch (E); placed the working channel (F); extracted the prolapse of nucleus pulposus (G); and sufficient decompression of the nerve root was seen under the endoscope $(\mathbf{H})$.
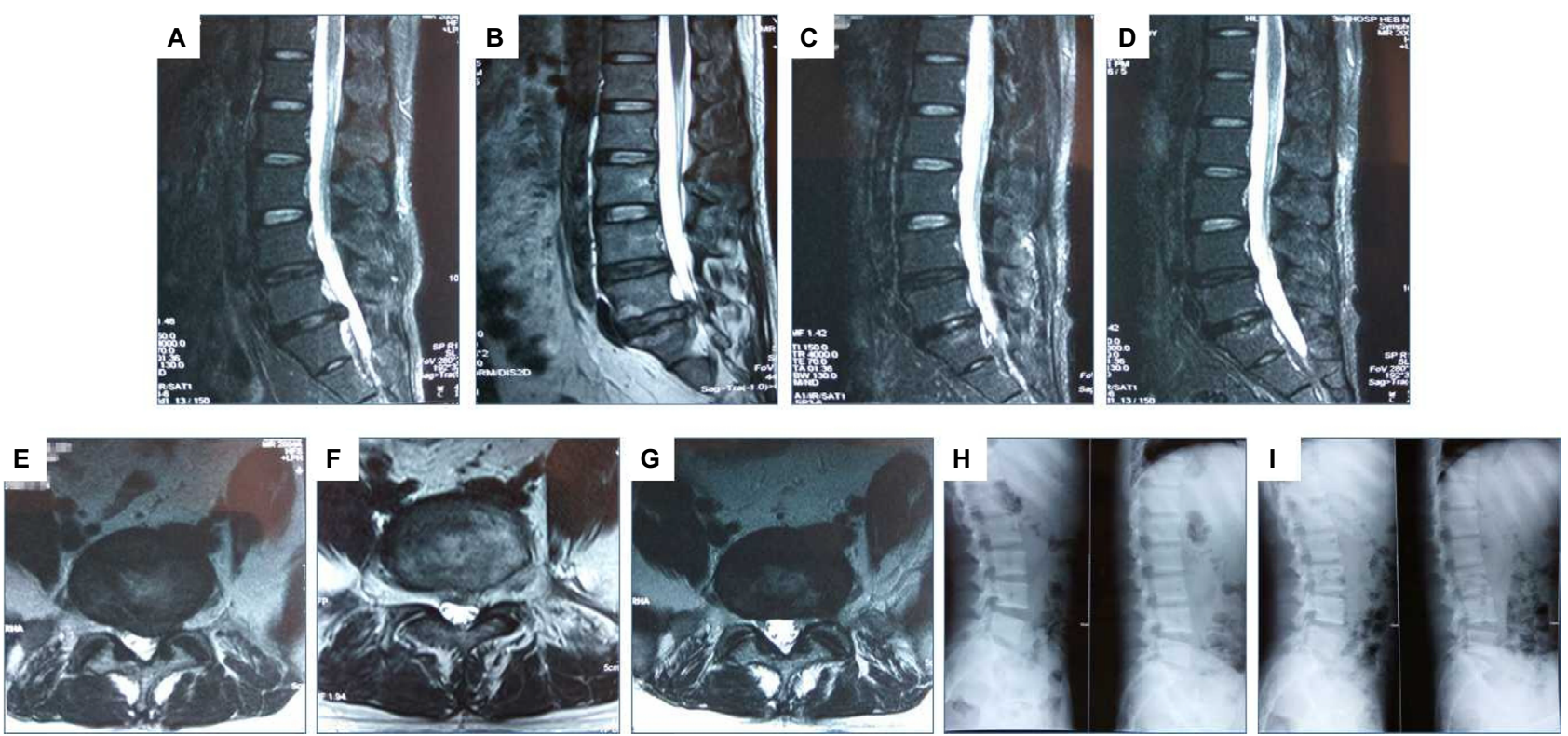

Figure 2 The MRI and X-ray changes.

Notes: Preoperative MRI in sagittal position (A); sagittal MRI immediately after surgery (B); sagittal MRI 3 months after surgery (C); sagittal MRI 24 months after surgery (D); preoperative MRI in coronal position (E); coronal MRI 3 months after surgery (F); coronal MRI 24 months after surgery (G); preoperative X-ray radiograph in lumbar dynamic position showing no lumbar instability or spondylolysis $(\mathbf{H})$; reexamination $\mathrm{X}$-ray radiograph in lumbar dynamic position 24 months postoperatively showing no lumbar instability (I).

Abbreviation: MRI, magnetic resonance imaging. 


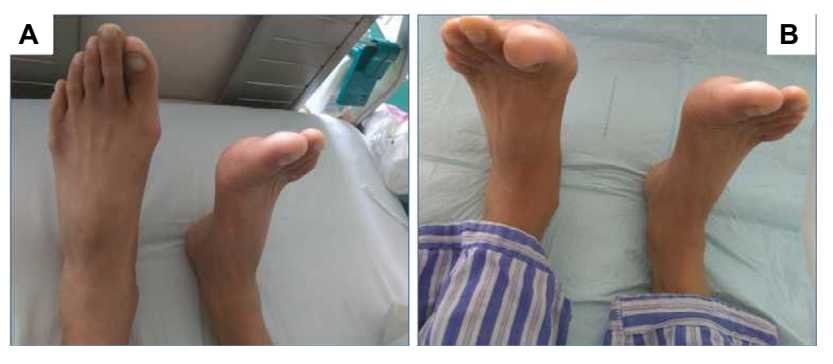

Figure 3 (A) Grade I preoperative anterior tibial muscle strength of the left lower extremity. (B) Grade 4 anterior tibial muscle strength of the left lower extremity immediately after the surgery.

\section{Discussion}

There are many reasons responsible for common peroneal nerve paralysis in clinical settings. Theoretically speaking, pathological changes in the neural structures that influence the dorsal stretch of ankle will induce common peroneal nerve paralysis, including trauma, external oppression, nerve compression, infection, tumor, and iatrogenic factors. ${ }^{15}$ Sometimes, the external pressure induced by the improper posture of crossing legs will also cause common peroneal nerve paralysis. Common peroneal nerve paralysis caused by lumbar diseases includes hematoma, ${ }^{16}$ ossification of the ligamentum flavum, ${ }^{17}$ discal cyst, ${ }^{18}$ zygapophyseal cyst, ${ }^{19}$ and epidural thrombosis. ${ }^{20}$ However, common peroneal nerve paralysis resulting from these reasons is rare in clinic, and it is mainly caused by degenerative lumbar diseases. In the studies of Iizuka et $\mathrm{al}^{4}$ and Jonsson et $\mathrm{al},{ }^{21} \mathrm{LDH}$ accounted for $57.0 \%$ of common peroneal nerve paralysis-associated lumbar diseases, whereas lumbar spinal stenosis accounted for only $43.0 \%$. Aono et $\mathrm{al}^{1}$ reported that $\mathrm{LDH}$ and lumbar spinal stenosis accounted for $52.0 \%$ and $35.0 \%$ of common peroneal nerve paralysis-associated lumbar diseases, respectively, while spondylolysis and spondylolisthesis were relatively rare causes of foot drop..$^{22}$ The typical manifestation of common peroneal nerve paralysis is foot drop, which may manifest as unilateral or bilateral, and bilateral foot drop is rare, which is mainly caused by lumbar spinal stenosis. Guigui et $\mathrm{al}^{22}$ reported in their study that bilateral foot drop accounted for $1 / 4$ of patients with lumbar spinal stenosis, regardless of the various degrees of bilateral anterior tibial muscle paralysis. In this study, no patients with bilateral foot drop were seen, which might be related to the fact that patients with lumbar spinal stenosis were not enrolled in the study.

The peroneal nerve that governs the anterior tibial muscle derives from the anterior horn cells of the low-lying spine, the axon of which enters the L4 and L5 nerve root and forms the lumbosacral trunk with other lumbosacral nerve roots together. These nerve fibers enter the lateral sciatic nerve trunk and form the peroneal nerve in the branch above the knee. Previous anatomical study discovered that the anterior tibial muscle is mainly governed by the $\mathrm{L} 4$ nerve root, ${ }^{23}$ but there are several reports regarding foot drop induced by L5 radiculopathy alone. Aono et $\mathrm{al}^{1}$ reported that among cases with foot drop induced by degenerative lumbar diseases, a majority of the lesion involved L4/5 segments. Therefore, it was speculated that foot drop might be mainly caused by L5 nerve root damage, and the anterior tibial muscle was mainly governed by $\mathrm{L} 5$ nerve root. The research by McCulloch et $\mathrm{al}^{24}$ demonstrated that L5 nerve root governed the anterior tibial muscle and the extensor hallucis longus, and the adjacent nerve roots $\mathrm{L} 4$ and $\mathrm{S} 1$ also governed these muscles. Iizuka et $\mathrm{al}^{4}$ suggested that the damage of two nerve roots (including L5) was the common mechanism for LDH-induced foot drop. In this study, five cases had L3/4 segment protrusion, 19 had L4/5, and eight had L5/S1 segment protrusion.

The conservative treatment of common peroneal nerve paralysis is mainly by wearing the pedorthic boots, which can prevent the occurrence of foot drop when the patient is walking. ${ }^{15}$ Though the nonsurgical treatments such as muscle relaxant, spinal cord manipulation, physiotherapy, and epidural steroid injection have been extensively applied in controlling the radiating pain, these methods cannot treat the decreased muscle strength. ${ }^{25}$ Foot drop is regarded as the sign of severe lumbar diseases, and almost all the reported cases are generally treated surgically. However, no clinical research aiming at surgical treatment of the LDH-induced foot drop has been reported so far. For the LDH-induced foot drop, a majority of surgeons consider that the foot drop should be treated as soon as possible once it occurs, so as to relieve the corresponding nerve compression. At present, the commonly used surgical method is the open lumbar posterior nerve root decompression and exploration. However, the traditional lumbar posterior open surgery requires extensive stripping and long-term traction of the bilateral paravertebral soft tissues, as well as the destruction of the posterior spinal bony structure, which frequently results in long-term postoperative lumbar back pain in some patients. Therefore, improving the therapeutic effects after the lumbar spinal surgery in patients, in the meantime, reducing the probability of occurrence of the postoperative complications is of great significance.

Compared with open surgery, TED has the advantages such as little trauma, little blood loss, rapid recovery, and little expense. ${ }^{9}$ However, application of TED in treating LDHinduced common peroneal nerve paralysis is only restricted in case report. ${ }^{10}$ There are no reports on the large sample size 
study aiming at applying such surgical method exclusively in treating LDH-induced common peroneal nerve paralysis, and hence its safety and effectiveness remain unclear. It is indicated in this study that $93.8 \%$ patients had the postoperative anterior tibial muscle strength recovered to $\geq$ grade 4 . Two patients who were evaluated as fair and poor in the modified $\mathrm{MacNab}$ therapeutic effect evaluation had poor postoperative effects, which might be related to the long preoperative course of disease of 16.3 and 12.5 months, respectively. It reminds us that the early or delayed surgery timing may influence the therapeutic effects of LDH-induced common peroneal nerve paralysis after TED, and the foot drop caused by LDH should be diagnosed and treated in the early stage. Though the motor function of the two cases in this research was not recovered in the last follow-up, the postoperative leg pain was markedly improved.

Currently, there is much controversy regarding the factors that influence the postoperative prognosis for degenerative lumbar disease-induced foot drop. A study which included 55 cases suggested that the age, etiology, course of disease, and preoperative anterior tibial muscle strength of patients had no obvious effects on the degree of postoperative recovery. ${ }^{3}$ The research by Aono et $\mathrm{al}^{1}(\mathrm{n}=46)$ revealed that the course of disease and preoperative muscle strength had significant influence on postoperative recovery. Some research also indicated that none of these factors showed no distinct influence on the postoperative recovery of patients with foot drop induced by $\mathrm{LDH}(\mathrm{n}=16)$, while the anterior tibial muscle strength and the extensor hallucis longus muscle strength had obvious influence on the postoperative recovery of patients with foot drop induced by lumbar spinal stenosis. Ghahreman et $\mathrm{al}^{2}$ reported in their study $(\mathrm{n}=56)$ that younger patients were associated with better postoperative recovery, while other factors showed no statistical difference on postoperative recovery. At present, there are huge differences among various research conclusions, which may be related to the small sample size and the selection bias of patients with different causes. Therefore, clinical research with large sample size is required in order to obtain more reliable clinical evidence.

In this study, three cases had the muscle strength recovered to grade 4 immediately after the surgery. The determinant for achieving such satisfactory surgical effects remained unknown, so it was considered that the key factor for successful surgery lies on sufficient nerve root decompression. Clinical experience reminds us that only by sufficiently expanding the intervertebral foramen can we guarantee the intraspinal mobility of the working channel, thus providing enough operating space for the displacement of the working channel toward the head, tail, ventral side, dorsal side, and the midline and consequently removing the protruding intervertebral disk. To cope with this issue, the following methods were adopted: 1) emphasized on target puncture, carefully analyzed the computed tomography and MRI images preoperatively and judged the site of herniation of intervertebral disk, namely, the site that the working channel terminal is placed eventually; 2) pressed the spinal needle close to the ventral side of the superior zygapophysis to the greatest extent in the lateral position, so that the zygapophyseal plasty could be better conducted; 3) the intervertebral foramen should be enlarged toward the dorsal side of the dural sac with the trephine consciously, so that more zygapophysis could be removed; on the other hand, prying and pressing when placing the working channel into the spine could gain more observational and decompression space; though severe zygapophysis destruction would induce increased lateral stretch range of motion of lumbar spine, it had no obvious effect on the lateral stretch and rotational stability, and the stability of the surgical segment in patients in this study was excellently maintained 2 years postoperatively; and 4 ) the working casing was rotated from the head to the tail during the surgery and moved along the nerve root to confirm that the dorsal side and the ventral side were sufficiently decompressed. The patients felt that the preoperative symptoms were apparently relieved and obvious nerve root pulsation could be seen under the endoscope, suggesting excellent angioplerosis, and thus the working channel and the endoscope could be removed.

One case in this study developed dural sac laceration intraoperatively, which might be attributed to the adhesion of the nerve root with the surrounding structure as a result of long course of disease in the patient. So, careful identification and patient separation should be conducted during operation under the endoscope, and blind clamping should be avoided to prevent accidental injury. One case developed abnormal sensation in export nerve root zone on the second day after the surgery, which might be related to the stimulation to the dorsal root ganglion of export nerve root during the process of placing the working channel as a result of the narrow intervertebral foramen of the patient. Therefore, for patients with narrow intervertebral foramen, attention should be paid to decompress the dorsal structure of the nerve root, so as to reduce the stimulation to the export nerve root.

\section{Conclusion}

TED can guarantee excellent intervertebral foramen enlargement and plasty and sufficiently decompress the nerve root; in parallel, it can obviously reduce surgical trauma and 
complications, and thus, it is a safe, effective, and minimally invasive treatment for patients with $\mathrm{LDH}$-induced common peroneal nerve paralysis. However, this research is based on only preliminary clinical results, and a long-term prospective clinical research with large sample size is required for further observation.

\section{Disclosure}

The authors report no conflicts of interest in this work.

\section{References}

1. Aono H, Iwasaki M, Ohwada T, et al. Surgical outcome of drop foot caused by degenerative lumbar diseases. Spine (Phila Pa 1976). 2007;32(8):E262-E266.

2. Ghahreman A, Ferch RD, Rao PJ, Bogduk N. Minimal access versus open posterior lumbar interbody fusion in the treatment of spondylolisthesis. Neurosurgery. 2010;66(2):296-304.

3. Girardi FP, Cammisa FJ, Huang RC, Parvataneni HK, Tsairis P. Improvement of preoperative foot drop after lumbar surgery. $J$ Spinal Disord Tech. 2002;15(6):490-494.

4. Iizuka Y, Iizuka H, Tsutsumi S, et al. Foot drop due to lumbar degenerative conditions: mechanism and prognostic factors in herniated nucleus pulposus and lumbar spinal stenosis. J Neurosurg Spine. 2009;10(3):260-264.

5. Rodriguez-Martinez NG, Perez-Orribo L, Kalb S, et al. The role of obesity in the biomechanics and radiological changes of the spine: an in vitro study. J Neurosurg Spine. 2016;24(4):615-623.

6. McGuire KJ, Khaleel MA, Rihn JA, Lurie JD, Zhao W, Weinstein JN. The effect of high obesity on outcomes of treatment for lumbar spinal conditions: subgroup analysis of the spine patient outcomes research trial. Spine (Phila Pa 1976). 2014;39(23):1975-1980.

7. Hu ZJ, Fang XQ, Zhou ZJ, Wang JY, Zhao FD, Fan SW. Effect and possible mechanism of muscle-splitting approach on multifidus muscle injury and atrophy after posterior lumbar spine surgery. $J$ Bone Joint Surg Am. 2013;95(24):e191-e192.

8. Choi KC, Kim JS, Park CK. Percutaneous endoscopic lumbar discectomy as an alternative to open lumbar microdiscectomy for large lumbar disc herniation. Pain Physician. 2016;19(2):E291-E300.

9. Wang YP, Zhang W, An JL, Zhang J, Bai JY, Sun YP. Evaluation of transforaminal endoscopic discectomy in treatment of obese patients with lumbar disc herniation. Med Sci Monit. 2016;22:2513-2519.
10. Chun EH, Park HS. A modified approach of percutaneous endoscopic lumbar discectomy (PELD) for far lateral disc herniation at L5-S1 with foot drop. Korean J Pain. 2016;29(1):57-61.

11. Ahn Y, Kim JU, Lee BH, et al. Postoperative retroperitoneal hematoma following transforaminal percutaneous endoscopic lumbar discectomy. J Neurosurg Spine. 2009;10(6):595-602.

12. Yeung AT, Yeung CA. Minimally invasive techniques for the management of lumbar disc herniation. Orthop Clin North Am. 2007;38(3):363-372.

13. Choi G, Kang HY, Modi HN, et al. Risk of developing seizure after percutaneous endoscopic lumbar discectomy. J Spinal Disord Tech. 2011;24(2):83-92.

14. Kang SH, Park SW. Symptomatic post-discectomy pseudocyst after endoscopic lumbar discectomy. J Korean Neurosurg Soc. 2011; 49(1):31-36.

15. Stewart JD. Foot drop: Where, why and what to do? Pract Neurol. 2008;8(3):158-169.

16. Kono H, Sasahara H, Furukawa S, Taguchi N. An analysis of background factors influencing selection of residency facility for dental students in universities located in rural areas in Japan. Open Dent J. 2015; 9:159-167.

17. Yano T, Doita M, Iguchi T, et al. Radiculopathy due to ossification of the yellow ligament at the lower lumbar spine. Spine (Phila Pa 1976). 2003;28(19):E401-E404.

18. Kim JU, Lee SH, Lee DY. Extraforaminal lumbar synovial cyst causing sudden foot drop: case report. Neurol Med Chir (Tokyo). 2008; 48(12):578-581.

19. Ghahreman A, Ferch RD, Rao P, Chandran N, Shadbolt B. Recovery of ankle dorsiflexion weakness following lumbar decompressive surgery. J Clin Neurosci. 2009;16(8):1024-1027.

20. Raynor RB, Saint-Louis L. Postoperative gas bubble foot drop. A case report. Spine (Phila Pa 1976). 1999;24(3):299-301.

21. Jonsson B, Stromqvist B. Motor affliction of the L5 nerve root in lumbar nerve root compression syndromes. Spine (Phila Pa 1976). 1995;20(18):2012-2015.

22. Guigui P, Benoist M, Delecourt C, Delhoume J, Deburge A. Motor deficit in lumbar spinal stenosis: a retrospective study of a series of 50 patients. J Spinal Disord. 1998;11(4):283-288.

23. Okuda S, Miyauchi A, Oda T, Haku T, Yamamoto T, Iwasaki M. Surgical complications of posterior lumbar interbody fusion with total facetectomy in 251 patients. $J$ Neurosurg Spine. 2006;4(4):304-309.

24. McCulloch JA, Waddell G. Variation of the lumbosacral myotomes with bony segmental anomalies. J Bone Joint Surg Br. 1980;62-B(4):475-480.

25. Sharma H, Lee SW, Cole AA. The management of weakness caused by lumbar and lumbosacral nerve root compression. J Bone Joint Surg Br. 2012;94(11):1442-1447.
Journal of Pain Research

\section{Publish your work in this journal}

The Journal of Pain Research is an international, peer reviewed, open access, online journal that welcomes laboratory and clinical findings in the fields of pain research and the prevention and management of pain. Original research, reviews, symposium reports, hypothesis formation and commentaries are all considered for publication.

\section{Dovepress}

The manuscript management system is completely online and includes a very quick and fair peer-review system, which is all easy to use. Visit http://www.dovepress.com/testimonials.php to read real quotes from published authors. 